\title{
Low cost and efficient synthesis of magnetic iron oxide/activated sericite nanocomposites for rapid removal of methylene blue and crystal violet dyes
}

\begin{abstract}
Activated sericite was used as a solid matrix for control over the size of iron oxide nanoparticles (NPs). The activation of sericite was achieved by undergoing thermal, acid and salt treatments. Different weight ratios (w.r.) of iron oxide to activated sericite were prepared by varying the amount of iron oxide. X-ray diffraction (XRD) patterns illustrated a decrease in the intensity of sericite peaks by increasing the amount of iron oxide. High resolution-transmission electron microscopy (HR-TEM) analysis showed the formation of highly distributed quasi-spherical iron oxide NPs with average diameter of about 3.56-7.78 nm. The formation of mesoporous iron oxide NPs on the surface and edge of the activated sericite surface were also observed under Field emission scanning electron microscopy (FESEM). Vibration sample magnetometer (VSM) measurement revealed the nanocomposites (NCs) with saturation magnetization of about 2.17$8.12 \mathrm{emu} \cdot \mathrm{g}-1$. Brunauer-Emmett-Teller (BET) surface area analysis revealed the specific surface area of NCs between 79.4 and $161.5 \mathrm{~m} 2 \cdot \mathrm{g}-1$. Fourier transform infrared (FTIR) spectra showed non-bond chemical interaction between the iron oxide and rigid silicate layers. Ultraviolet-visible (UV-vis) diffuse reflectance spectroscopy confirms that the material has band gap energy of about $2.55 \mathrm{eV}$. The $\mathrm{NC}$ with w.r. of 1:3 (iron oxide to activated sericite) showed the best performance with high specific surface area and sufficient magnetization for easy magnetic separation. The selected NC showed maximum removal efficiency over $99 \%$ for methylene blue and crystal violet dyes within $20 \mathrm{~min}$.
\end{abstract}

Keyword: Activated sericite; Iron oxide; Nanoparticles; Nanocomposites; Dyes removal; Adsorption 